Volume 23 (2018) 25-41

DOI: $10.24330 /$ ieja.373634

\title{
ELEMENTARY RADICAL CLASSES
}

\author{
B. J. Gardner \\ Received: 16 April 2016; Revised: 29 November 2017; Accepted: 04 December 2017 \\ Communicated by Abdullah Harmancı
}

\begin{abstract}
A radical class $\mathcal{R}$ of rings is elementary if it contains precisely those rings whose singly generated subrings are in $\mathcal{R}$. Many examples of elementary radical classes are presented, and all those which are either contained in the Jacobson radical class or disjoint from it are described. Attention is given to those elementary radical classes which are definable by composition subsemigroups of the free ring on one generator. Whether every elementary radical class is of this form remains an open question.
\end{abstract}

Mathematics Subject Classification (2010): 16N80, 08C99

Keywords: Radical class, locally equational, Mal'tsev-Neumann product

\section{Introduction}

A radical class $\mathcal{R}$ is elementary if it satisfies the condition

$A \in \mathcal{R}$ if and only if every singly generated subring of $A$ is in $\mathcal{R}$.

Such classes have been considered by several authors under several names. They were called elementary by Stewart [25], (though in his systematic terminology they were called 2-radical classes), semi-strictly hereditary (polustrogo nasledstvennye) by Ryabukhin [23] and 1-radical classes by the author [9].

All rings considered (apart from some remarks at the end) are associative. The terminology and notation of radical theory generally follow [13], but we use script capitals rather than lower case Greek letters for classes of rings. A class is strongly hereditary if it contains all subrings of its members. The free ring on a single generator $x$ is called $F$. The polynomial ring over a $\operatorname{ring} A$ is called $A[X]$, even when $A=\mathbb{Z}$, but we shall have occasion to identify $F$ with $X \mathbb{Z}[X]$ and accordingly to conflate $X$ and $x$. The subring generated by a ring element $a$ is called $\langle a\rangle$, while the subsemigroup generated by a semigroup element $f$ is called $[f]$. The ring of integers modulo $n$ is denoted by $\mathbb{Z}_{n}$, a cyclic group of order $n$ by $\mathbb{Z}(n)$ and a field with $n$ elements by $K(n) . R^{+}$denotes the additive group of a ring $R, G^{0}$ or $R^{0}$ the zeroring on an abelian group $G$ or the additive group of a ring $R$ respectively. 
The variety generated by a ring $A$ or a set $M$ of equations is called $V A R(A)$ or $V A R(M)$ respectively.

For an element $a$ of a ring $A$ we have a homomorphism $\alpha: F \rightarrow A$ with $\alpha(x)=a$. If $f=k_{1} x+k_{2} x^{2}+\cdots+k_{n} x^{n} \in F$, then $\alpha(f)=k_{1} a+k_{2} a^{2}+\cdots+k_{n} a^{n}$, and we will call this $f(a)$. Then the image of $\alpha$ is $\{f(a): f \in F\}$, i.e. the subring $\langle a\rangle$ generated by $a$, and its kernel is $\{f: f(a)=0\}$. Let $(0 * a)=\{f: f(a)=0\}$, so that $\langle a\rangle \cong F /(0 * a)$. There is a strong resemblance here to a description of a singly generated submodule as isomorphic to a factor module of the scalar ring. This similarity no doubt provided at least some motivation for our next concept.

A radical filter of ideals of $F$ is a non-empty set $\Phi$ of ideals satisfying the following conditions.

If $I \in \Phi$ and $I \subseteq J$, then $J \in \Phi$.

If $J \in \Phi$, then $(0 *(f+J)) \in \Phi$ for all $f \in F$.

If $J \in \Phi, I \triangleleft F, I \subseteq J$ and $(0 *(f+I)) \in \Phi$ for all $f \in J$, then $I \in \Phi$.

Theorem 1.1. (Ryabukhin [23]) Radical filters and elementary radical classes determine each other uniquely by the inverse correspondences

$$
\begin{gathered}
\Phi \mapsto\{A:(0 * a) \in \Phi \forall a \in A\} ; \\
\mathcal{R} \mapsto\{(0 * a): a \in A \in \mathcal{R}\} .
\end{gathered}
$$

This is analogous to Gabriel's result [8] linking idempotent topologising filters of left ideals of a ring with identity and hereditary radical classes (= hereditary torsion classes $=$ strongly complete Serre classes etc.) of unital left modules. The parallel between radical classes of rings and modules is particularly strong in the case of elementary ring radicals.

There is another way in which we can use $F$ to get elementary radical classes. Our definition of $f(a), f \in F, a$ a ring element, gives us a meaning for $f(g)$ where $f, g \in F$. Writing $f \circ g$ instead of $f(g)$, we get a binary operation with respect to which $F$ is a semigroup. For a o-subsemigroup $S$ of $F$ let

$$
\mathcal{R}_{S}=\{A: \forall a \in A \exists f \in S ; f(a)=0\} .
$$

Theorem 1.2. For each o-subsemigroup $S$ of $F, \mathcal{R}_{S}$ is an elementary radical class.

Proof. Clearly $\mathcal{R}$ is homomorphically closed and if $I$ is the union of an ascending chain of ideals of some ring, all of which are in $\mathcal{R}_{S}$, then $I \in R$. If $J \triangleleft A$, and if $J$ and $A / J$ are in $\mathcal{R}_{S}$, let $a$ be an element of $A$. Then for some $g \in S$ we have $g(a+J)=0$, i.e. $g(a) \in J$. But then there is an $f \in S$ such that $f \circ g(a)=f(g(a))=0$. Hence $A \in \mathcal{R}_{S}$. It follows that $\mathcal{R}_{S}$ is a radical class. Fairly clearly a ring $R$ is in $\mathcal{R}_{S}$ if and only if each singly generated subring is in $\mathcal{R}_{S}$. 
We will mention just two examples for now.

Example 1.3. If $S=\left\{x^{n}: n \in \mathbb{Z}^{+}\right\}$then $\mathcal{R}_{S}=\mathcal{N}$, the nil radical class.

Example 1.4. If $S=\left\{n x: n \in \mathbb{Z}^{+}\right\}$then $\mathcal{R}_{S}=\mathcal{T}$, the class of torsion rings, i.e. rings with torsion additive group.

We find such "semigroup representations" for many elementary radical classes, and present evidence which suggests that semi-simple radical classes (which are elementary radical classes) do not have such representations.

One class which does have such a representation is the class of extensions of torsion rings by nil rings, i.e. the Mal'tsev-Neumann product $\mathcal{T} \circ \mathcal{N}$. This leads

to an investigation of the question "When is the Mal'tsev-Neumann product of (elementary) radical classes a(n) (elementary) radical class?". Among other things it will be shown in [11] that a Mal'tsev-Neumann product of elementary radical classes need not be a radical class, and even when it is it need not be elementary.

Elementary radical classes are examples of locally equational classes as defined by $\mathrm{Hu}$ [15]. The latter are generalizations of varieties, so since Mal'tsev-Neumann products of varieties are varieties, there may be some interest in the result that products of locally equational classes need not be locally equational. This follows from results on products of radical classes in [11].

\section{Examples of elementary radical classes defined by semigroups}

We begin with a list of polynomial conditions for ring elements.

A ring element is

(i) algebraic if it satisfies an equation

$$
x^{m}+a_{1} x^{m+1}+a_{2} x^{n+2}+\cdots+a_{k} x^{m+k}=0, a_{i} \in \mathbb{Z} ;
$$

(ii) integral if it satisfies an equation

$$
a_{1} x+a_{2} x^{2}+\cdots+a_{n-1} x^{n-1}+x^{n}=0, a_{i} \in \mathbb{Z} ;
$$

(iii) strongly integral if it satisfies an equation

$$
a_{1} x+a_{2} x^{2}+\cdots+a_{n-1} x^{n-1}+x^{n}=0, a_{i} \in \mathbb{Z}, a_{1} \neq 0 ;
$$

(iv) constrained if it satisfies an equation

$$
\begin{gathered}
a_{k} x^{k}+a_{k+1} x^{k+1}+\cdots+a_{m-1} x^{m-1}+a_{m} x^{m}=0, a_{i} \in \mathbb{Z}, k<m, \text { some } \\
a_{i} \neq 0 ;
\end{gathered}
$$

(v) anti-integral if it satisfies an equation

$$
x+a_{1} x+a_{2} x^{2}+\cdots+a_{n} x^{n}=0, a_{i} \in \mathbb{Z} ;
$$

(vi) periodic if it satisfies an equation $x^{n}-x=0, n>1$; 
(vii) weakly periodic if it satisfies an equation $x^{m}-x^{n}=0, m>n \geq 1$.

A ring will be said to have one of these properties precisely when each of its elements satisfies the appropriate definition.

Each property defines an elementary radical class. This is reasonably clear for (i)-(v) as the polynomials of the type occurring in the prescribed equations form o-semigroups. For (vi) and (vii) we need the following results.

Theorem 2.1. (Szász [26], Stewart [24], Osborn [21]) A ring is periodic if and only if it is anti-integral.

Theorem 2.2. (Drazin [7], Chacron [5]) A ring is weakly periodic if and only if it is algebraic.

The class of periodic (= anti-integral) rings is called $\mathcal{B}_{1}$ in [24]. We shall extend this terminology for the radical classes just introduced.

Example 2.3. The following are elementary radical classes.

(i) $\mathcal{B}_{1}$ : the class of periodic (= anti-integral) rings.

(ii) $\mathcal{B}_{2}$ : the class of weakly periodic (= algebraic) rings.

(iii) $\mathcal{B}_{3}$ : the class of integral rings.

(iv) $\mathcal{B}_{4}$ : the class of constrained rings.

(v) $\mathcal{B}_{5}$ : the class of strongly integral rings.

We have already mentioned the elementary radical classes $\mathcal{N}$ of nil rings and $\mathcal{T}$ of torsion rings. For a prime $p$ we denote by $\mathcal{T}_{p}$ the class of rings whose additive groups are $p$-groups which on occasion we will call $p$-rings (though noting that this term has been used with a different meaning in parts of the literature). Since intersections of elementary radical classes are elementary radical classes, we now have a reasonable collection. The next result describes some containment relations.

Theorem 2.4. We have the following containments.

(i) $\mathcal{B}_{1} \subsetneq \mathcal{B}_{2} \subsetneq \mathcal{B}_{3} \subsetneq \mathcal{B}_{4}$.

(ii) $\mathcal{B}_{1} \subseteq \mathcal{B}_{5}$.

(iii) $\mathcal{B}_{2} \nsubseteq \mathcal{B}_{5}$.

(iv) $\mathcal{B}_{5} \subsetneq \mathcal{B}_{3}$.

(v) $\mathcal{B}_{2} \cap \mathcal{T} \subsetneq \mathcal{B}_{5}$.

(vi) $\mathcal{N} \subseteq \mathcal{B}_{2}$ and $\mathcal{B}_{1} \cap \mathcal{N}=\{0\}$.

(vii) $\mathcal{B}_{1} \subsetneq \mathcal{T}$.

(viii) $\mathcal{B}_{2} \nsubseteq \mathcal{T}$. 
There are no containments involving these radical classes besides (i)-(viii) and their obvious consequences.

Proof. (i) Clearly $\mathcal{B}_{1} \subseteq \mathcal{B}_{2} \subseteq \mathcal{B}_{3} \subseteq \mathcal{B}_{4}$ (if we use the anti-integral and algebraic properties). All nil rings are in $\mathcal{B}_{2}$ (as if $a^{n}=0$ then $a^{n}=a^{n+1}$ ) but $\mathcal{B}_{1}$ contains no non-zero ones. If $m \in \mathbb{Z}$ then $(-2 m) 2 m+(2 m)^{2}=0$, so $2 \mathbb{Z} \in \mathcal{B}_{3}$. But $2 \mathbb{Z}$ is not weakly periodic, i.e. $2 \mathbb{Z} \notin \mathcal{B}_{2}$. All torsion rings are in $\mathcal{B}_{4}$, but, e.g., $K(p)[X] \notin \mathcal{B}_{3}$, since $X$ does not satisfy a polynomial equation of the appropriate kind.

(ii) This is clear.

(iii) All nil rings are in $\mathcal{B}_{2}$ but, e.g., the zero ring $\mathbb{Z}^{0}$ on the integers is not in $\mathbb{B}_{5}$.

(iv) Clearly $\mathcal{B}_{5} \subseteq \mathcal{B}_{3}$, but by (i) and (iii) $\mathcal{B}_{3} \nsubseteq \mathcal{B}_{5}$.

(v) If $b \in A \in \mathcal{B}_{2} \cap \mathcal{T}$ and $b \neq 0$, then $b^{m}=b^{n}$ for some $m, n \in \mathbb{Z}^{+}$with $m<n$. Let $k$ be the order of $b$. Then $b$ satisfies $-k x-x^{m}+x^{n}=0$. Hence $A \in \mathcal{B}_{5}$. But the argument in (i) actually shows that $2 \mathbb{Z} \in \mathbb{B}_{5}$ and it is not in $\mathcal{T}$.

(vi) As noted in (iii), $\mathcal{N} \subseteq \mathcal{B}_{2}$. There are no non-trivial nil periodic rings.

(vii) By Theorem 1 of [26], Theorem 3.4 of [24] and Theorem 13.2 of [21], $\mathcal{B}_{1} \subseteq \mathcal{T}$. But torsion nil rings are not in $\mathcal{B}_{1}$.

(viii) As we have seen, $\mathcal{N} \subseteq \mathcal{B}_{2}$, and certainly $\mathcal{N} \nsubseteq \mathcal{T}$.

Note that $\mathcal{B}_{4}$ is the largest non-trivial elementary radical class (see [23]).

We shall see further examples of semigroups and the elementary radical classes they define, but we break in here to prove a theorem that not only provides an important part of the classification of elementary radical classes but is needed for one of our semigroup examples. For a non-empty set $E$ of primes, let $\mathcal{T}_{E}$ denote the class of rings whose additive groups are direct sums of $p$-groups, $p \in E$. (So if $E=\{p\}$ then $\mathcal{T}_{E}=\mathcal{T}_{p}$ as defined before, and when $E$ contains all the primes $\mathcal{T}_{E}=\mathcal{T}$.)

Theorem 2.5. The proper elementary radical subclasses of $\mathcal{N}$ are precisely the classes $\mathcal{N} \cap \mathcal{T}_{E}$ for non-empty sets $E$ of primes.

Proof. Clearly the $\mathcal{T}_{E}$ are elementary radical classes, so the $\mathcal{N} \cap \mathcal{T}_{E}$ are also. Let $\mathcal{U}$ be an elementary radical class with $\{0\} \subsetneq \mathcal{U} \subsetneq \mathcal{N}$. Let $E=\{p: p$ is a prime and $\left.\mathcal{U} \cap \mathcal{T}_{p} \neq\{0\}\right\}$. If $0 \neq a \in A \in \mathcal{U}$, then $\langle a>$ is nilpotent and $\langle a\rangle \in \mathcal{U}$. Now $\langle a\rangle /<a\rangle^{2}$ is in $\mathcal{U}$ and is the zeroring on the cyclic group generated by $a+\langle a\rangle^{2}$. If this is finite it has a subring isomorphic to the zeroring $\mathbb{Z}(p)^{0}$ on the cyclic group of order $p$ for some prime $p$. If it is infinite it has all rings $\mathbb{Z}(p)^{0}$ as homomorphic images. In any case, $\mathcal{U}$ contains $\mathbb{Z}(p)^{0}$ for some prime $p$, so $E \neq \emptyset$. Let $B$ be a non-zero $p$-ring in $\mathcal{U}$ for any $p \in E$. If $0 \neq b \in B$ the subring 
$\langle b\rangle$ is a nilpotent ring in $\mathcal{U}$ so the zeroring $\langle b\rangle^{+0}$ is also in $\mathcal{U}$ ([10], p.84, 5.6 Corollary). Hence $\mathcal{U}$ contains the zeroring $\mathbb{Z}(p)^{0}$ on the cyclic group of order $p$, and consequently all nilpotent $p$-rings. If now $R$ is any nil $p$-ring, then all its singly generated subrings are in $\mathcal{U}$, so $R$ is also. We conclude that $\mathcal{N} \cap \mathcal{T}_{p} \subseteq \mathcal{U}$. This being so for every $p \in E$, it follows that $\mathcal{N} \cap \mathcal{T}_{E} \subseteq \mathcal{U}$. Suppose $\mathcal{U}$ contains a torsion-free ring $C$ with a non-zero element $c$. Then arguing as with $p$-rings above, we see that $\mathcal{U}$ contains the zeroring $\mathbb{Z}^{0}$ on the integers, all nilpotent rings and hence all nil rings, contrary to our stipulation. Thus $\mathcal{U}$ only contains torsion rings, and clearly these are in $\mathcal{N} \cap \mathcal{T}_{E}$.

The Chebyshev polynomials of the first kind, usually called $T_{0}, T_{1}, T_{2}, \ldots$, form a well known semigroup with interesting properties. Their coefficients are integers, $T_{n}$ has degree $n$ for each $n$ and we have $T_{m} \circ T_{n}=T_{m n}$ for all $m, n$. The Chebyshev polynomials of the second kind will not be considered, so in the sequel we shall generally suppress "of the first kind" and just refer to "Chebyshev polynomials". For an account of these polynomials see Rivlin [22]. We will just demonstrate a few facts that will be needed.

For each $n$ we have $T_{n}(\cos \theta)=\cos n \theta, 0 \leq \theta \leq \pi$, so $T_{n}(0)=\cos n \frac{\pi}{2}$. If $n$ is odd then the $x$-free term of $T_{n}$ is $T_{n}(0)=0$, so $T_{n} \in F$. Thus the Chebyshev polynomials of odd degree form a o-subsemigroup of $F$ and therefore define an elementary radical class. (When $n$ is even the $x$-free term of $T_{n}$ is \pm 1 , so $T_{n} \notin F$.)

By formula 1.10, p.4 of [22], the coefficient of $x$ in $T_{2 n+1}$ is $(-1)^{n}\left(\begin{array}{c}2 n+1 \\ 2 n\end{array}\right)=$ $(-1)^{n}(2 n+1)$. For each $n$ we have $T_{n}(1)=\operatorname{cosn} \cos ^{-1} 1=\cos n 0=1$, so the sum of the coefficients of $T_{n}$ is 1 .

Theorem 2.6. When $S$ is the set of Chebyshev polynomials of the first kind with odd degree, $\mathcal{R}_{S}$ is the class of odd-torsion nil rings.

Proof. Suppose first that $A$ is a torsion-free ring in $\mathcal{R}_{S}$. If $a \in A \backslash\{0\}$ and $T_{n}(a)=$ 0 , let

$$
T_{n}=a_{1} x+a_{2} x^{2}+\cdots+a_{n} x^{n}
$$

Then $a_{1} a=-a_{2} a^{2}-\cdots-a_{n} a^{n}$. Passing to the divisible hull $\mathbb{Q} \otimes A$, since $a_{i}=$ $\pm n \neq 0$, we can write $a=-\frac{a_{2}}{a_{1}} a^{2}-\cdots-\frac{a_{n}}{a_{1}} a^{n}$. Let $e=-\frac{a_{2}}{a_{1}} a-\cdots-\frac{a_{n}}{a_{1}} a^{n-1}$. Then $e a=a=a e$, whence $e e=e$. Since this idempotent $e$ is in $\mathbb{Q} \otimes A$, for some $m \in \mathbb{Z}^{+}$ we have $m e \in A$. Then $(m e)^{k}=m^{k-1} m e$ for all $k \in \mathbb{Z}^{+}$. Since $m e \in A$ we have $T_{r}(m e)=0$ for some odd $r$. Let $T_{r}=b_{1} x+b_{2} x^{2}+\cdots+b_{r} x^{r}$. Then 


$$
\begin{gathered}
0=T_{r}(m e)=b_{1} m e+b_{2}(m e)^{2}+\cdots+b_{r}(m e)^{r}= \\
b_{1} m e+b_{2} m \cdot m e+\cdots+b_{r} m^{r-1} \cdot m e=\left(b_{1}+b_{2} m+\cdots+b_{r} m^{r-1}\right) m e .
\end{gathered}
$$

But $m e \neq 0($ as $e a=a \neq 0)$ and $\mathrm{A}$ is torsion-free, so $b_{1}+b_{2} m+\cdots+b_{r} m^{r-1}=0$, whence

$$
T_{r}(m)=\left(b_{1}+b_{2} m+\cdots+b_{r} m^{r-1}\right) m=0
$$

But $T_{r}$ has no integer zeros apart from $0([22]$, p.6, 1.17) so there can be no such element $a$. Thus there are no non-zero torsion-free rings in $\mathcal{R}_{S}$, which class, being homomorphically closed, consists of torsion rings.

We shall next show that $\mathcal{R}_{S}$ contains only nil rings. Suppose there is a ring $R \in \mathcal{R}_{S}$ with $\mathcal{J}(R) \neq R$, where $\mathcal{J}(R)$ is the Jacobson radical. Then since $\mathcal{R}_{S}$ is homomorphically closed, it contains a primitive ring, and being strongly hereditary must contain a matrix ring over a division ring (see, e.e. [14], pp.43-44), the division ring itself, its centre and finally the prime subfield of the centre. As $\mathcal{R}_{S}$ contains only torsion rings, we conclude that $K(p) \in \mathcal{R}_{S}$ for some prime $p$. Let $i_{p}$ denote the identity of $K(p)$. Then for some odd $s$ we have $T_{s}\left(i_{p}\right)=0$. But then, if $T_{s}=c_{1} x+\cdots+c_{s} x^{s}$, we have

$$
0=T_{s}\left(i_{p}\right)=c_{1} i_{p}+\cdots+c_{s} i_{p}^{s}=\left(c_{1}+\cdots+c_{s}\right) i_{p}=i_{p}
$$

(as the sum of the coefficients is 1). From this contradiction we conclude that there is no such $R$, so that $\mathcal{R}_{S} \subseteq \mathcal{J}$. But all subrings of rings in $\mathcal{R}_{S}$ are themselves in $\mathcal{R}_{S}$ and hence in $\mathcal{J}$. It follows that $\mathcal{R}_{S} \subseteq \mathcal{N}[27]$.

Now suppose there exists a $b \in B \in \mathcal{R}_{S}$ with $2 b=0 \neq b$. Let $T_{u}(b)=0$ where $u$ is odd and $T_{u}=d_{1} x+\cdots+d_{u} x^{u}$. Then $d_{1}= \pm u \neq 0$ and $d_{1}$ is odd. Let $d_{1}=2 w+1$. Then $b=\left(d_{1}-2 w\right) b=d_{1} b=-d_{2} b^{2}-\cdots-d_{u} b^{u}\left(\operatorname{as} T_{u}(b)=0\right)$. Hence $-d_{2} b-\cdots-d_{u} b^{u-1}$ is an identity for the subring $<b>$. But $B$ is nil, so this identity is zero and $b=0$, contrary to our assumption. Thus all rings in $\mathcal{R}_{S}$ are 2-torsion-free.

We have thus far shown that every ring in $\mathcal{R}_{S}$ is an odd-torsion nil ring. The converse is quick and easy. For a prime $p$, let $\mathbb{Z}(p)^{0}$ be the zero ring on a cyclic group of order $p$. If $p$ is odd then for each $z \in \mathbb{Z}(p)^{0}$ we have $T_{p}(z)= \pm p z+($ terms in higher powers of $z)=0$, so $\mathbb{Z}(p)^{0} \in \mathcal{R}_{S}$. But then by Theorem $2.5 \mathcal{N} \cap \mathcal{T}_{p} \subseteq \mathcal{R}_{S}$ (for all odd primes $p$ ).

Corollary 2.7. If $p$ is an odd prime and $V$ is the semigroup of Chebyshev polynomials $T_{p^{n}}$ then $\mathcal{R}_{V}$ is the class of nil p-rings. 
Proof. By Theorem 2.6 $\mathcal{R}_{V}$ is contained in the class of odd-torsion nil rings. If $a \in A \in \mathcal{R}_{V}$ then $\langle a\rangle \in \mathcal{R}_{V}$ so $\langle a\rangle^{+0} \in \mathcal{R}_{V}$. Hence for "the $a$ in $\langle a\rangle^{+0}$ " there exists an $n \in \mathbb{Z}^{+}$such that

$$
0=T_{p^{n}}(a)= \pm p^{n} a+(\text { terms in higher powers of } a)= \pm p^{n} a .
$$

Hence $A \in \mathcal{T}_{p}$, so $\mathcal{R}_{V} \subseteq \mathcal{N} \cap \mathcal{T}_{p}$. Conversely, if $B \neq 0$ is a $p$-ring with zero multiplication, $b \in B$ and $b$ has order $p^{t}$, then $T_{p^{t}}(b)= \pm p^{t} b+$ (terms in higher powers of $b)=0$, so $B \in \mathcal{R}_{V}$. It now follows from Theorem 2.5 that $\mathcal{R}_{V}=\mathcal{N} \cap$ $\mathcal{T}_{p}$

A complete commuting family of real polynomials is a set of polynomials, at least one for each degree $\geq 0$, which commute under composition. The polynomials $X^{n}$ form one such family, and so do the Chebyshev polynomials $T_{n}$. These are actually "almost" the only ones: a linear polynomial $\lambda=a X+b, a \neq 0$ has a o-inverse $\frac{1}{a} X-\frac{b}{a}$ which we will call $\lambda^{-1}$. Clearly (using loose terminology) the $\lambda^{-1} \circ X^{n} \circ \lambda$ and the $\lambda^{-1} \circ T_{n} \circ \lambda$ also form complete commuting families. It happens that every such family arises in one of these two ways [4], [17]. All of this happens in $\mathbb{R}[X]$; it is rare for one of these conjugations to take $\mathbb{Z}[X]$ to $\mathbb{Z}[X]$, and rarer yet to take $F$ (which we can obviously identify with $X \mathbb{Z}[X]$ ) to itself. It does happen though.

Example 2.8. For $\lambda=X+1$, we have $\lambda^{-1}=X-1$, and conjugation produces subsemigroups of $F$ from the set of power polynomials and the set of all (not necessarily of odd degree) Chebyshev polynomials. For conjugation produces, for any polynomial $\alpha$, the polynomial given by

$$
\lambda^{-1} \circ \alpha \circ \lambda(X)=\alpha(X+1)-1 .
$$

Thus

$$
\lambda^{-1} \circ X^{n} \circ \lambda(X)=(X+1)^{n}-1=X^{n}+n X^{n-1}+\cdots+n X \in F .
$$

Also, If $T_{n}=a_{0}+a_{1} X+a_{2} X^{2}+\cdots+a_{n} X^{n}$, then

$T_{n}(X+1)$

$=a_{0}+a_{1}(X+1)+a_{2}\left(X^{2}+2 X+1\right)+\cdots+a_{n}\left(X^{n}+n X^{n-1}+\left(\begin{array}{c}n \\ 2\end{array}\right) X^{n-2}+\cdots+n X+1\right)$

$=a_{0}+a_{1}+\cdots+a_{n}+($ terms in $X)=1+($ terms in $X)$, so $T_{n}(X+1)-1 \in F$.

Note that if we use $X-1$ instead of $X+1$ we do not get subsemigroups of $F$ from either of the complete families. However this conjugation does work on the $T_{n}$ for odd $n$ : the $X$-free term of $T_{n}(X-1)$ is -1 since the coefficients of all even powers of $X$ in $T_{n}$ are zero. 
The polynomials $(X+1)^{n}-1$ (or $(x+1)^{n}-1$, reverting to our previous notation for $F$ ), define one of our well known elementary radical classes for which we have not yet seen a representing semigroup. (The "1" in $(x+1)^{n}$ can be viewed as an adjoined identity element or a calculating device; it is not "really there".)

Theorem 2.9. Let $S=\left\{(x+1)^{n}-1: n \in \mathbb{Z}^{+}\right\}$. Then $\mathcal{R}_{S}$ is the class of all torsion nil rings.

Proof. First we note another way of describing the polynomials $(x+1)^{n}-1$. Since the symbol $\circ$ is being used for composition, we will use $*$ for the operation of quasiregularity: $a * b=a+b+a b$. Since $*$ is associative, we can refer unambiguously to the "*-powers": $a^{* n}=a * a * a \cdots * a(n$ symbols $a)$. For $n>1$ we have $(x+1)^{n}-1=x^{* n}$; this is easily proved by induction.

If $a \in A \in \mathcal{R}_{S}$ and $a \neq 0$, then for some $n>1$ we have $a * a^{*(n-1)}=a^{* n}=0$ (and of course $0 * 0=0$ ). Thus every element $a$ is quasiregular in the subring $\langle a\rangle$ which it generates. From this it follows that every subring of $A$ is quasiregular, so by the result from [27] already used, $A$ is nil. Thus $\mathcal{R}_{S} \subseteq \mathcal{N}$. Since $(x+1)^{n}-1=$ $x^{n}+\cdots+\left(\begin{array}{c}n \\ 2\end{array}\right) x^{2}+n x$, it is clear that the zeroring $\mathbb{Z}^{0}$ on the integers is not in $\mathcal{R}_{S}$. But for each prime $p$, the zeroring $\mathbb{Z}(p)^{0}$ on the cyclic group of order $p$ satisfies the equation $(x+1)^{p}-1=0$, so $\mathbb{Z}(p)^{0} \in \mathcal{R}_{S}$. It now follows from Theorem 2.5 that $\mathcal{R}_{S}=\mathcal{N} \cap \mathcal{T}$.

Corollary 2.10. For each prime $p$ let $S_{p}=\left\{(x+1)^{p^{n}}-1: n \in \mathbb{Z}^{+}\right\}$. Then $\mathcal{R}_{S_{p}}=\mathcal{N} \cap \mathcal{T}_{p}$

Proof. As we saw in the proof of Theorem 2.9, the zeroring $\mathbb{Z}(p)^{0}$ satisfies the identity $(x+1)^{p}-1=0$, so

$$
\mathcal{N} \cap \mathcal{T}_{p} \subseteq \mathcal{R}_{S_{p}} \subseteq \mathcal{N} \cap \mathcal{T} .
$$

By Theorem 2.5, all we need prove, therefore, is that $\mathcal{R}_{S_{p}}$ contains no ring $\mathbb{Z}(q)^{0}$ for any prime $q \neq p$. Suppose $\mathbb{Z}(q)^{0}$ is in $\mathcal{R}_{S_{p}}$ and let $b$ be its generator. Then for some $n$ we have $(b+1)^{p^{n}}=0$, whence $p^{n} b=-\left(b^{p^{n}}+p^{n} b^{p^{n}-1}+\cdots+\left(\begin{array}{c}p^{n} \\ 2\end{array}\right) b^{2}\right)=0$, so $q=p$.

Note. Corollary 2.10 applies in particular when $p=2$; previously we only had a semigroup representation for $\mathcal{N} \cap \mathcal{T}_{p}$ for odd $p$ (Corollary 2.7).

Corollary 2.11. Let $A$ be a quasiregular ring, $p$ a prime. The circle group of $A$ is a torsion group (resp. a p-group) if and only if $A$ is nil and its additive group is a torsion group (resp. a p-group). 
Corollary 2.12. (Amberg and Dickenschied [1], Lemma 2.4) Let $A$ be a nil ring, $p$ a prime. Then the circle group of $A$ is a p-group if and only if its additive group is a p-group.

Conjugation by $X+1$ transforms the Chebyshev polynomials into a subsemigroup of $F$. Clearly this applies also to subsemigroups of the $T_{n}$ (and here we mean subsemigroups in $F$, as even $n$ is allowed). In particular we can take the $T_{2^{n}}, n \in$ $\mathbb{Z}^{+}$.

Example 2.13. The polynomials $T_{2^{n}}(x+1)-1$ form a $\circ$-subsemigroup $S$ of $F$. The corresponding $\mathcal{R}_{S}$ contains all rings $A$ for which $2 A=0$, since they satisfy

$$
0=2 x^{2}+4 x=2\left(x^{2}+2 x+1\right)-2=\left(2(x+1)^{2}-1\right)-1=T_{2}(x+1)-1 .
$$

In particular it contains the zeroring $\mathbb{Z}(2)^{0}$ and hence all nil 2-rings.

Let $R$ be a 2-ring. Then if $r \in R$ and $2^{n} r=0$, we have $(2 r)^{n}=0$, so $2 R$ is a nil ring and hence is in $\mathcal{R}_{S}$. But also $2(R / 2 R)=0$, so $R / 2 R \in \mathcal{R}_{S}$, whence $R \in \mathcal{R}_{S}$, so that $\mathcal{T}_{2} \subseteq \mathcal{R}_{S}$. But this inclusion is proper. For instance $T_{4}(x+1)-1$

$=\left(8(x+1)^{4}-8(x+1)^{2}+1\right)-1$

$=8\left(x^{4}+4 x^{3}+6 x^{2}+4 x+1\right)-8\left(x^{2}+2 x+1\right)+1-1$

$=8 x^{4}+32 x^{3}+40 x^{2}+16 x$.

In a ring $B$ with $3 B=0$, this is equivalent to $2 x^{4}+2 x^{3}+x^{2}+x$, which is satisfied by all rings in $\operatorname{VAR}\left(x^{3}=x, 3 x=0\right)$, and in particular by $K(3)$.

We will look at one more family of semigroups defining elementary radical classes. The radical classes they define exemplify the following notion.

If $\mathcal{R}$ and $\mathcal{U}$ are radical classes (not necessarily elementary), their Mal'tsevNeumann product [19], [20], is the class

$$
\mathcal{R} \circ \mathcal{U}=\{R: \exists I \triangleleft R ; I \in \mathcal{R} ; R / I \in \mathcal{U}\} .
$$

Rather this is one of their Mal'tsev products; there is also $\mathcal{U} \circ \mathcal{R}$. Not much is known as to which Mal'tsev products of radical classes are themselves radical classes. We shall have a bit more to say about this in [11]. The following simple result is useful.

Proposition 2.14. Let $\mathcal{R}$ and $\mathcal{U}$ be radical classes. $A$ ring $A$ is in $\mathcal{R} \circ \mathcal{U}$ if and only if $A / \mathcal{R}(A) \in \mathcal{U}$.

Let $P$ be a set of primes, $P^{*}$ the submonoid of $\mathbb{Z}^{+}$generated by $P$ and let

$$
S_{P}=\left\{m x^{n}: m \in P^{*}, n \in \mathbb{Z}^{+}\right\} .
$$

Then as $m x^{n} \circ k x^{\ell}=m\left(k x^{\ell}\right)^{n}=m k^{n} x^{\ell n} \in S_{P}$ for all $m, k \in P^{*}, n, \ell \in \mathbb{Z}^{+}, S_{P}$ is a subsemigroup of $F$. 
Theorem 2.15. For every set $P$ of primes, we have $\mathcal{R}_{S_{P}}=\mathcal{T}_{P} \circ \mathcal{N}$.

Proof. If $A \in \mathcal{R}_{S_{P}}$ then $A / \mathcal{T}_{P}(A) \in \mathcal{R}_{S_{P}}$, so for each $a \in A$ there exist $m \in$ $P^{*}, n \in \mathbb{Z}^{+}$such that $m\left(a+\mathcal{T}_{P}(A)\right)^{n}=0$ and hence $\left(a+\mathcal{T}_{P}(A)\right)^{n}=0$. Thus $A / \mathcal{T}_{P}(A)$ is nil, i.e. $A$ is in $\mathcal{T}_{P} \circ \mathcal{N}$. Conversely, if $b \in B \in \mathcal{T}_{P} \circ \mathcal{N}$, then for some $\ell \in \mathbb{Z}^{+}$we have $b^{\ell}+\mathcal{T}_{P}(B)=\left(b+\mathcal{T}_{P}(B)\right)^{\ell}=0$, i.e. $b^{\ell} \in \mathcal{T}_{P}(B)$. But then for some $k \in P^{*}$ we have $k b^{\ell}=0$. Hence $B \in \mathcal{R}_{S_{P}}$.

In particular, when $P$ is the set of all primes, $\mathcal{R}_{S_{P}}=\mathcal{T} \circ \mathcal{N}$ and when $P=\emptyset$, $\mathcal{R}_{S_{P}}=\mathcal{N}$.

\section{Are all elementary radical classes defined by semigroups?}

The non-trivial semi-simple radical classes (hereafter SSR-classes), are the varieties generated by finite sets of finite fields ([24] or [13], Section 3.20). As noted in [25], they are elementary radical classes. Perhaps the easiest way to see this is to observe that as varieties they are defined by identities in one variable [12].

We do not have an example of an elementary radical class not definable by a subsemigroup of $F$. On the other hand, we do not have a semigroup which represents an $S S R$-class. One semigroup associated with an $S S R$-class is the (one variable) $T$-ideal defining it as a variety, but this defines an enormous radical class (see Theorem 3.3 below). When the $S S R$-class $\mathcal{V}$ is defined by a single identity $f=0$, we can also consider the semigroup $[f]$ generated by $f$, but the radical class $\mathcal{R}_{[f]}$ is still much bigger than $\mathcal{V}$, at least sometimes (see below).

Let $\mathfrak{B}$ denote the $S S R$-class of boolean rings, defined as a variety by $K(2)$ and by the identity $x-x^{2}=0$. We will consider the semigroup $\left[x-x^{2}\right]$ generated by $x-x^{2}$. We have to consider powers in semigroups such as this with respect to $\circ$. To avoid confusion with the multiplication of $F$, we will indicate "o-powers" by notation such as $f^{\circ \text { n. For instance }}$

$$
\left(x-x^{2}\right)^{\circ 2}=x-x^{2}-\left(x^{2}-2 x^{3}+x^{4}\right)=x-2 x^{2}+2 x^{3}-x^{4} .
$$

In what follows we will need to consider different elements $f, g$ of $F$ which have the same effect when applied to the elements of a ring $A$. (For example $2 x^{4}+2 x^{3}+x^{2}+x$ has the same effect as $x^{2}+x$ (or $x^{2}-x$ ) in any ring $A$ with $2 A=0$, and the same effect as 0 in $K(2)$.) We will write

$$
f \sim g \text { in } A
$$

to indicate this.

Proposition 3.1. In every ring $A$ with $2 A=0,\left(x-x^{2}\right)^{\circ 2^{n}} \sim x-x^{2^{\left(2^{n}\right)}}$ for all $n$. 
Proof. $\left(x-x^{2}\right)^{\circ 2^{1}}=x-x^{2}-\left(x-x^{2}\right)^{2} \sim x-x^{2}-x^{2}-x^{4} \sim x-x^{4}=x-x^{2^{2^{1}}}$. If $\left(x-x^{2}\right)^{\circ 2^{k}} \sim x-x^{2^{\left(2^{k}\right)}}$ then $\left(x-x^{2}\right)^{\circ 2^{k+1}}=\left(x-x^{2}\right)^{\circ 2^{k} \cdot 2}=\left(x-x^{2}\right)^{\circ 2^{k}} \circ\left(x-x^{2}\right)^{\circ 2^{k}} \sim\left(x-x^{2^{\left(2^{k}\right)}}\right) \circ\left(x-x^{2^{\left(2^{k}\right)}}\right)=$ $\left(x-x^{2^{\left(2^{k}\right)}}\right)-\left(x-x^{2^{\left(2^{k}\right)}}\right)^{2^{\left(2^{k}\right)}} \sim x-x^{2^{\left(2^{k}\right)}}-x^{2^{\left(2^{k}\right)}}-\left(x^{2^{\left(2^{k}\right)}}\right)^{2^{\left(2^{k}\right)}} \sim x-\left(x^{2^{\left(2^{k}\right)}}\right)^{2^{\left(2^{k}\right)}}=$ $x-x^{2^{\left(2^{k}+2^{k}\right)}}=x-x^{2^{\left(2^{k+1}\right)}}$. The result follows by induction.

For $f \in F$, let $[f]$ denote the o-semigroup generated by $f$. Now $\mathfrak{B}$ is defined by $x^{2}-x$ as well as $x-x^{2}$, but the radical classes $\mathcal{R}_{\left[x-x^{2}\right]}$ and $\mathcal{R}_{\left[x^{2}-x\right]}$ are different.

Theorem 3.2. (i) $\left\{A \in \mathcal{R}_{\left[x-x^{2}\right]}: 2 A=0\right\}=\left\{A \in \mathcal{R}_{\left[x^{2}-x\right]}: 2 A=0\right\}=$ $\left\{A \in \mathcal{B}_{1}: 2 A=0\right\}$.

(ii) $K(3) \in \mathcal{R}_{\left[x-x^{2}\right]}$.

(iii) $K(3) \notin \mathcal{R}_{\left[x^{2}-x\right]}$.

Proof. (i) If $a \in A \in \mathcal{R}_{\left[x-x^{2}\right]}$ and $\left(a-a^{2}\right)^{\circ n}=0$, then $\left(a-a^{2}\right)^{\circ(n+1)}=(a-$ $\left.a^{2}\right)^{\circ n}-\left(\left(a-a^{2}\right)^{\circ n}\right)^{2}=0$ and so on, so all higher powers of $x-x^{2}$ vanish at $a$.

For each $m \in \mathbb{Z}^{+}$there is a $k$ with $2^{m} \leq 2^{\left(2^{k}\right)}$. But then $K\left(2^{m}\right) \subseteq K\left(2^{\left(2^{k}\right)}\right)$ so $K\left(2^{m}\right)$ satisfies $x-x^{2^{\left(2^{k}\right)}} \sim\left(x-x^{2}\right)^{\circ 2^{k}}$ by Proposition 3.1 Thus $K\left(2^{m}\right) \in \mathcal{R}_{\left[x-x^{2}\right]}$. Since $\mathcal{R}_{\left[x-x^{2}\right]}$ contains all finite fields of characteristic 2 , it contains all rings $B \in \mathcal{B}_{1}$ for which $2 B=0$, by Theorem 3.4 of [24] or Theorem 1 of [26]. The reverse containment is clear from Theorem 2.1.

The proof for $\mathcal{R}_{\left[x^{2}-x\right]}$ is similar.

(ii) In $K(3)$ we have $\left(x-x^{2}\right)^{\circ 2}=\left(x-x^{2}\right)-\left(x-x^{2}\right)^{2}=x-2 x^{2}+2 x^{3}-x^{4} \sim$ $x-2 x^{2}+2 x-x^{2}=3 x-3 x^{2} \sim 0$.

(iii) In $K(3)$ we have $\left(x^{2}-x\right)^{\circ 2}=x^{4}-2 x^{3}+x \sim x^{2}-2 x+x=x^{2}-x$. Thus all iterates of $x^{2}-x$ have the same effect in $K(3)$, and since $2^{2}-2 \neq 0$ there, $K(3) \notin \mathcal{R}_{\left[x^{2}-x\right]}$.

We shall not follow this line further, beyond observing that $K(5)$ is in both $\mathcal{R}_{\left[x-x^{3}\right]}$ and $\mathcal{R}_{\left[x^{3}-x\right]}($ as is $K(2))$.

Let $\mathbb{B}$ denote the one-variable $T$-ideal of $F$ generated by $x-x^{2}$ (or $x^{2}-x$ ). Then $\mathbb{B}$ is closed under endomorphisms of $F$. For all this, see [21], p.173. Thus if $f, g \in \mathbb{B}$, then for the endomorphism for which $x \mapsto g$ we have $f \mapsto f \circ g$, so $f \circ g \in \mathbb{B}$. Thus $\mathbb{B}$ is a o-semigroup; its elementary radical is the largest proper elementary radical class.

Theorem 3.3. $\mathcal{R}_{\mathbb{B}}=\mathcal{B}_{4}$.

Proof. Since $\left((2 x)^{2}-2 x\right)-4\left(x^{2}-x\right)=2 x$ we have $2 x \in \mathbb{B}$ and hence $2 n x \in \mathbb{B}$ for all $n \in \mathbb{Z}$. If $a \in A \in \mathcal{B}_{4}$, then $b_{1} a+b_{2} a^{2}+\cdots+b_{k} a^{k}=0$ for some $b_{1}, b_{2}, \ldots, b_{k} \in \mathbb{Z}$, 
not all $=0$. Hence $2 b_{1} a+2 b_{2} a^{2}+\cdots+2 b_{k} a^{k}=0$. But each $2 b_{i} x \in \mathbb{B}$, so each $b_{i} x^{i}=2 b_{i} x \cdot x^{i-1} \in \mathbb{B}$ for $i>1$ and finally $b_{1} x+b_{2} x^{2}+\cdots+b_{k} x^{k} \in \mathbb{B}$, so $A \in \mathcal{R}_{\mathbb{B}}$. This proves that $\mathcal{B}_{4} \subseteq \mathcal{R}_{\mathbb{B}}$. On the other hand, $F \notin \mathcal{R}_{\mathbb{B}}$ so the latter is not the class of all rings.

\section{Closing remarks}

We have now accumulated a substantial collection of elementary radical classes and eliminated some plausible candidates. How close are we to a complete classification? The next result provides a little information.

In what follows, $\mathcal{J}$ is the Jacobson radical class.

Theorem 4.1. $\quad$ (i) Let $\mathcal{R}$ be an elementary radical class.

(a) If $\mathcal{R} \subseteq \mathcal{J}$, then $\mathcal{R} \subseteq \mathcal{N}$.

(b) If $\mathcal{R} \cap \mathcal{J}=\{0\}$, then $\mathcal{R} \subseteq \mathcal{B}_{1}$.

(ii) The radical subclasses of $\mathcal{N}$ are, $\{0\}, \mathcal{N}$ and the $\mathcal{N} \cap \mathcal{T}_{E}, E \neq \emptyset$.

(iii) The elementary radical subclasses of $\mathcal{B}_{1}$ are determined by the sets of fields they contain.

Proof. (i) (a) If $A \in \mathcal{R} \subseteq \mathcal{J}$ then every subring of $A$ is in $\mathcal{J}$ so $A$ is nil [27].

(b) If $\mathcal{R} \cap \mathcal{J}=\{0\}$ and $R \in \mathcal{R}$ then $\mathcal{J}(R)=0$. Thus $\mathcal{R}$ is contained in the class of rings, each homomorphic image of which is $\mathcal{J}$-semi-simple. If $A \in \mathcal{R}$ then so is every primitive image $\bar{A}$. Since $\mathcal{R}$ is strongly hereditary and contains no quasiregular rings, $\bar{A}$ must be a division ring (see [14], Theorem 2.1.4, pp. 43-44). Let $K$ be the centre of $\bar{A}$. Then $K \in \mathcal{R}$. If the characteristic of $K$ were 0 then $\mathcal{R}$ would contain $\mathbb{Q}, 2 \mathbb{Z}$ and hence $2 \mathbb{Z} / 4 \mathbb{Z}$, while the last-named is quasiregular. Hence $K$ has prime characteristic $p$. If $\bar{A}$ contains an element $u$ which is transcendental over the prime subfield $K(p)$ of $\bar{A}$, then $\mathcal{R}$ contains $\langle u\rangle$ and all of its homomorphic images, some of which are nilpotent. From this contradiction we conclude that $\bar{A}$ is algebraic over $K(p)$ and hence commutative (Jacobson [16], Theorem 8). We have now shown that every primitive homomorphic image of $A$ is an algebraic field extension of some $K(p)$. Hence $A$ is commutative. But also every homomorphic image of $A$ is semiprime (being $\mathcal{J}$-semi-simple) and therefore $A$ is hereditarily idempotent (see [2] or [6]). The combination of these last two facts yields the result that $A$ is (strongly) regular ([3], Theorem 1 and Remark (1) following it). Since $\mathcal{R}$ is strongly hereditary, it therefore consists of rings of which every subring is (strongly) regular, so by Theorem 1 of $[26], \mathcal{R} \subseteq \mathcal{B}_{1}$.

(ii) This is Theorem 2.5. 
(iii) Let $\mathcal{R}$ be an elementary radical subclass of $\mathcal{B}_{1}, \mathcal{F}_{\mathcal{R}}$ the class of fields in $\mathcal{R}$. (Up to isomorphism it has $2^{\aleph_{0}}$ members, corresponding to the subfields of algebraic closures of the fields $K(p)$ which are in $\mathcal{R}$.) Then $\mathcal{F}_{\mathcal{R}}$ is strongly hereditary. If $K \in \mathcal{F}_{\mathcal{R}}$ and $K$ has characteristic $p$, then we can assume that $K(p) \subseteq K \subseteq K(p)^{*}$, where $K(p)^{*}$ is an algebraic closure of $K(p)$. Thus we have the following description of $\mathcal{F}_{\mathcal{R}}$. For each prime $q$ let $\mathcal{F}_{\mathcal{R}}^{(q)}$ denote the class of fields in $\mathcal{F}_{\mathcal{R}}$ with characteristic q. Then $\mathcal{F}_{\mathcal{R}}=\bigcup_{q} \mathcal{F}_{\mathcal{R}}^{(q)}$

Now suppose $\mathcal{R}, \mathcal{U}$ are elementary radical subclasses of $\mathcal{B}_{1}$ with $\mathcal{F}_{\mathcal{R}}=\mathcal{F}_{\mathcal{U}}$. If $a \in A \in \mathcal{R}$ then $\langle a\rangle \in \mathcal{R}$ and so $\langle a\rangle$ is a direct sum of finitely many fields, which must be in $\mathcal{R}$ (see [26], Theorem 1 or [24], Theorem 3.4). But these fields are also in $\mathcal{U}$, so $\langle a>\in \mathcal{U}$. This being so for every $a \in A$, we see that $A \in \mathcal{U}$ and $\mathcal{R} \subseteq \mathcal{U}$. In the same way, $\mathcal{U} \subseteq \mathcal{R}$.

The $S S R$-classes are the elementary radical classes $\mathcal{R}$ for which

(i) $K(q)^{*} \notin \mathcal{F}_{\mathcal{R}}^{(q)}$ for all $q$ and

(ii) $\mathcal{F}_{\mathcal{R}}^{(q)}=\emptyset$ for almost all $q$.

Theorem 4.1 does not come close to providing a complete catalogue. In particular, it is not true that $\mathcal{R}=(\mathcal{R} \cap \mathcal{N}) \circ\left(\mathcal{R} \cap \mathcal{B}_{1}\right)$ for every $\mathcal{R}$.

Example 4.2. We have $\mathcal{N}, \mathcal{B}_{1} \subseteq \mathcal{B}_{2}$, but $\mathcal{B}_{2} \neq \mathcal{N} \circ \mathcal{B}_{1}$. For instance $M_{2}(K(2)) \in$ $\operatorname{VAR}\left(x^{2}=x^{8}\right) \subseteq \mathcal{B}_{2}$. (This is tedious but straightforward to verify. For a description of all the periodic identities satisfied by matrix rings over finite fields in general, see [18].) But $M_{2}(K(2))$ is simple and is neither in $\mathcal{N}$ nor $\mathcal{B}_{1}$, so it is not in $\mathcal{N} \circ \mathcal{B}_{1}$.

Since intersections of elementary radical classes are elementary radical classes, we can get a few, though not many, further examples from those we have mentioned by taking intersections.

We finally point out a few more containment results.

Theorem 4.3. We have the following containments between elementary radical classes.

(i) $\mathcal{B}_{2} \subsetneq \mathcal{T} \circ \mathcal{N}$.

(ii) $\mathcal{T} \circ \mathcal{N} \nsubseteq \mathcal{B}_{3}$.

(iii) $\mathcal{B}_{3} \nsubseteq \mathcal{T} \circ \mathcal{N}$.

(iv) $\mathcal{T} \circ \mathcal{N} \subsetneq \mathcal{B}_{4}$

Proof. (i) and (ii) If $A \in \mathcal{B}_{2}$, then $A / \mathcal{T}(A)$ is algebraic and torsion-free and hence nil (Drazin [7], Corollary 3.1, Chacron [5], Propositions 1 and 2), i.e. $A \in \mathcal{T} \circ \mathcal{N}$, 
but, e.g., $K(p)[X] \in \mathcal{T} \backslash \mathcal{B}_{3}$ for every prime $p$, so $\mathcal{T} \circ \mathcal{N} \nsubseteq \mathcal{B}_{3}$ and hence $\mathcal{T} \circ \mathcal{N} \nsubseteq \mathcal{B}_{2}$. (iii) As noted in the proof of Theorem 2.4, $2 \mathbb{Z} \in \mathcal{B}_{3}$; clearly it is not in $\mathcal{T} \circ \mathcal{N}$.

(iv) Like every non-trivial elementary radical class, $\mathcal{T} \circ \mathcal{N} \subseteq \mathcal{B}_{4}$. But, e.g, every algebraic field extension of $\mathbb{Q}$ is in $\mathcal{B}_{4}$ but not in $\mathcal{T} \circ \mathcal{N}$.

Although we have confined our attention to associative rings, the same sorts of questions can be asked for rings of other kinds, groups and so on. Note that since the one-generator associative free ring $F$ is also a free power-associative ring, so results based on radical filters or subsemigroups of $F$ go over verbatim to powerassociative rings.

We close with three open questions.

(1) Is every elementary radical class definable by a subsemigroup of $F$ ? In particular are all (or any?) elementary radical subclasses of $\mathcal{B}_{1}$ so definable?

(2) Let $\mathcal{R}$ be an elementary radical subclass of $\mathcal{B}_{1}, \mathcal{U}$ the upper radical class defined by the set of fields in $\mathcal{R}$. What is the relationship between $\mathcal{R}$ and the class of strongly $\mathcal{U}$-semi-simple rings? [2] (An answer to this question would go nicely with Stewart's result [25] that supernilpotent elementary radical classes are all special.)

(3) For each $S S R$-classes $\mathcal{V}$ the product $\mathcal{N} \circ \mathcal{V}$ is a radical class. [11] For which $\mathcal{V}$ is it elementary?

Acknowledgment. The author thanks Elena Cojuhari for tracking down a copy of [23].

\section{References}

[1] B. Amberg and O. Dickenschied, On the adjoint group of a radical ring, Canad. Math. Bull., 38(3) (1995), 262-270.

[2] V. A. Andrunakievič, Radicals of associative rings I, Amer. Math. Soc. Transl. (Ser. 2), 52 (1966), 95-128. [Russian original: Mat. Sb. N.S., 44(86) (1958), 179-212.]

[3] E. P. Armendariz and J. W. Fisher, Regular P.I.-rings, Proc. Amer. Math. Soc., 39 (1973), 247-251.

[4] H. D. Block and H. P. Thielman, Commutative polynomials, Quart. J. Math., Oxford Ser. (2), 2 (1951), 241-243.

[5] M. Chacron, On a theorem of Herstein, Canad. J. Math., 21 (1969), 1348-1353.

[6] R. C. Courter, Rings all of whose factor rings are semi-prime, Canad. Math. Bull., 12 (1969), 417-426. 
[7] M. P. Drazin, Algebraic and diagonable rings, Canad. J. Math., 8 (1956), 341354.

[8] P. Gabriel, Des catégories abeliennes, Bull. Soc. Math. France, 90 (1962), 323448.

[9] B. J. Gardner, Radical properties defined locally by polynomial identities, I, J. Austral. Math. Soc. Ser. A, 27(3) (1979), 257-273.

[10] B. J. Gardner, Radical Theory, Pitman Research Notes in Mathematics Series, 198, Longman Scientific \& Technical, Harlow; copublished in the United States with John Wiley \& Sons, Inc., New York, 1989.

[11] B. J. Gardner, A note on Mal'tsev-Neumann products of radical classes, Int. Electron. J. Algebra, to appear.

[12] B. J. Gardner and P. N. Stewart, On semisimple radical classes, Bull. Austral. Math. Soc., 13(3) (1975), 349-353.

[13] B. J. Gardner and R. Wiegandt, Radical Theory of Rings, Monographs and Textbooks in Pure and Applied Mathematics, 261, Marcel Dekker, Inc., New York, 2004.

[14] I. N. Herstein, Noncommutative Rings, The Carus Mathematical Monographs, 15, Published by The Mathematical Association of America; distributed by John Wiley \& Sons, Inc., New York, 1968.

[15] T. K. Hu, Locally equational classes of universal algebras, Chinese J. Math., 1(2) (1973), 143-165.

[16] N. Jacobson, Structure theory for algebraic algebras of bounded degree, Ann. of Math., 46 (1945), 695-707.

[17] E. Jacobsthal, Über vertauschbare polynome, Math. Z., 63 (1955), 243-276.

[18] A. A. Klein, On Fermat's theorem for matrices and the periodic identities of $M_{n}(G F(q))$, Arch. Math. (Basel), 34(5) (1980), 399-402.

[19] A. I. Mal'tsev, Multiplication of classes of algebraic systems, Russian, Sibirsk. Mat. Zh., 8 (1967), 346-365.

[20] H. Neumann, Varieties of Groups, Springer-Verlag, New York, Inc., New York, 1967.

[21] J. M. Osborn, Varieties of algebras, Advances in Math., 8 (1972), 163-369.

[22] T. J. Rivlin, Chebyshev Polynomials, From approximation theory to algebra and number theory, Second edition, Pure and Applied Mathematics, John Wiley \& Sons, Inc., New York, 1990. 
[23] Yu. M. Ryabukhin, Semistrictly hereditary radicals in primitive classes of rings, Russian, Studies in General Algebra (Sem.) (Russian), Akad. Nauk Moldav. SSR, Kishinev, (1965), 111-122.

[24] P. N. Stewart, Semi-simple radical classes, Pacific J. Math., 32 (1970), 249-254.

[25] P. N. Stewart, Strongly hereditary radical classes, J. London Math. Soc. (2), 4 (1972), 499-509.

[26] F. Szász, A class of regular rings, Monatsh. Math., 75 (1971), 168-172.

[27] W. J. Wickless, A characterization of the nil radical of a ring, Pacific J. Math., 35 (1970), 255-258.

\section{B. J. Gardner}

Discipline of Mathematics

University of Tasmania

PB 37, Hobart, TAS 7001

Australia

e-mail: Barry.Gardner@utas.edu.au 